\title{
Biomarcadores de estrés laboral en residentes: artículo de revisión
}

\section{Biomarkers of labor stress in residents: review article}

\author{
Jina Paola Burbano Obando ${ }^{1}$, Juan Pablo Sánchez Sánchez ${ }^{2}$, Andry Yasmid Mera Mamian³
}

\section{RESUMEN}

A traués del tiempo, se han generado múltiples definiciones para estrés, pero aún no se logra un consenso satisfactorio; en general, es percibido como una amenaza que pone en riesgo la armonía interna. Esta condición genera como respuesta una serie de cambios fisiológicos (respuesta adaptativa) que buscan mantener el equilibrio; con la activación subyacente de ejes como el hipotálamo hipófisis- suprarrenal, cuyas sustancias liberadas tienen

\begin{abstract}
Across the years, multiple definitions for stress have been generated, but a satisfactory consensus has not yet been reached; in general, it isperceived as a threat that risks the internal harmony. This condition generates in response a series of physiological changes (adaptive response) that seek to maintain the balance; with the underlying activation of axes such as the hypothalamus-pituitaryadrenal, whose released substances have an impact on
\end{abstract}

\section{Historial del artículo:}

Fecha de recepción: 07/09/2018

Fecha de aprobación: 18/04/2019

1 Citosalud. Médica especialista en Anatomía patológica, Manizales, Colombia.

2 Universidad del Cauca. Médico especialista en Anatomía patológica. Popayán, Colombia

3 Universidad CES, Fisioterapeuta, Magister en epidemiología en servicios de salud, estudiante de Doctorado en Epidemiología y Bioestadística, Medellín (Colombia).

Correspondencia: Jina Paola Burbano. Dirección: Carrera 53 \#14c-129, apartamento G502, Conjunto Gratamira L, Barrio el Limonar, Cali, Valle. Telefono: 3102528385. Correo Electrónico: jinaburbano@hotmail.com

Como citar este artículo: Burbano-Obando J, Sánchez JP, Mera-Mamian A. Biomarcadores de estrés laboral en residentes: artículo de revisión. Revista de la Facultad de Ciencias de la Salud de la Universidad del Cauca. 2019; 21 (2) 24-31 
impacto en varios sistemas, lo que explicaría el desarrollo deciertas enfermedadesyjustifica el usodebiomarcadores para la medición objetiva del estrés. Los médicos residentes en formación se han identificado como una población en riesgo para presentar estrés, específicamente estrés laboral o burnout (en inglés), el cual es posible medir de manera subjetiva mediante la aplicación de cuestionarios como el Maslach Burnout Inventory Human Services Survey (MBI), y a través del cual se ha identificado alta prevalencia de la condición; sin embargo, aún no existe consenso respecto a las estrategias de medición objetiva. La presente revisión pretende explorar la literatura existente sobre los biomarcadores como herramientas de medición de estrés laboral en residentes.

Palabras clave: Estrés laboral. Citoquinas. Educación Médica de Posgrado. Cortisol. Biomarcadores. (DeCS)

\section{INTRODUCCIÓN}

En el ańo 1997, Selye definió el estrés como un estado de homeostasis amenazada, que se reestablece mediante un complejo repertorio de respuestas adaptativas fisiológicas y conductuales del organismo (1). Por su parte Lazarus (2006), seńala que consiste en un proceso dinámico que ocurre cuando un individuo evalúa las demandas situacionales como el exceso de recursos disponibles (2). Otros autores se refieren al estrés como cualquier estímulo que pueda ser una amenaza para el bienestar físico o psicológico de un individuo y pone a prueba su capacidad de afrontamiento, por lo que se considera también un mecanismo de adaptación $(3,4)$.

En la investigación clínica y epidemiológica se cuenta con múltiples herramientas para medir el estrés psicológico; evaluando la respuesta afectiva personal a las demandas 0 mediante la medición de productos de los ejes fisiológicos activados tras la exposición a agentes estresores (5). Existen escalas como la de Ansiedad, la Depresión y Estrés (DASS21) (6), la Escala de estrés percibido (EEP) que evalúa el estrés emocional (7) y el Maslach Burnout Inventory-Human Services Survey (MBI-HSS), para la medición del estrés laboral (8).

El proceso de adaptación desencadenado bajo situaciones de estrés involucra cambios fisiológicos, los cuales requieren la activación del sistema neuroendocrino que además tiene influencias sobre diversos sistemas como el inmunológico $(1,9)$; ante este escenario algunos autores han descrito cambios en la concentración de algunos several systems, which would explain the development of some diseases and justify the use of biomarkers for the objective measurement of stress. The resident physicians in training have been identified as a population at risk for presenting stress, specifically job stress or burnout, which is possible to measure subjetively by applying questionnaires such as the Maslach Burnout Inventory Human Services Survey (MBI), which demonstrated a high prevalence of this condition; however, there is not a consensus yet regarding objective measurement strategies. The present review aims to explore the available literature on biomarkers as tools for measuring job stress in residents.

Key words: Burnout. Cytokines. Education, Medical, Graduate. Hydrocortisone. Biomarkers. (MeSH)

biomarcadores como: el cortisol, interleuquina 6 (IL-6) (10), antagonista del receptor de interleuquina 1 (IL-1 ra), interleuquina 10 (IL-10) (11), factor de necrosis tumoral alfa (TNF- $\alpha$ ) e interleuquina 2 (IL-2) (12).

Entre las poblaciones en riesgo para el desarrollo de estrés laboral se encuentran los residentes, quienes experimentan el trabajo como uno de los estresores principales (13). Las residencias médicas consisten en un modelo de aprendizaje basado principalmente en el componente asistencial con un promedio de 32 horas laborales semanales, a lo que se suman actividades académicas; según Sarmiento esta es una de las razones por la que los médicos residentes se consideran estudiantes y trabajadores (14). Se ha descrito que esta población es especialmente susceptible de padecer estrés laboral; aunque la prevalencia varía entre el $17.6 \%$ a $76 \%$ dependiendo de las cohortes estudiadas; variables como el ambiente laboral competitivo y la deprivación del sueńo favorecen el desarrollo de esta condición $(15,16)$, que a su vez se ha relacionado con el inicio de eventos desfavorables en salud para los residentes como lo son la aparición de cefalea, alteraciones visuales, problemas digestivos, cervicalgia, dorsalgia, irritabilidad y trastorno depresivo mayor, todo esto con consecuencias sobre la salud del propio individuo y repercusiones sobre el paciente por el incremento del error en la atención médica $(13,17,18)$.

La evaluación subjetiva del estrés en este tipo de poblaciones ha sido ampliamente descrita, pero se ha identificado un vacío relacionado con las estrategias de medición objetiva. Otras revisiones, exploran las alteraciones de 
varios biomarcadores relacionadas con estrés laboral en diferentes tipos de empleos como banqueros, oficinistas, taxistas, enfermeras o universitarios, de modo que la presente revisión pretende exponer la información existente enfocándose solamente en la población de médicos especialistas en formación (19-21).

Este artículo de revisión se enfoca en estrés laboral y la medición objetiva de este en médicos residentes. Primero se conceptualiza el estrés según diversas teorías, así como algunas causas relacionadas, luego se explican los cambios fisiológicos desencadenados por eventos estresantes a nivel del eje hipotálamo-hipófisis suprarrenal y los biomarcadores implicados, se enfatiza en el trabajo y situaciones implicadas en el desarrollo de estrés. Posteriormente se expone cómo los médicos especialistas durante su formación están expuestos a condiciones laborales estresantes, se mencionan las herramientas disponibles para la evaluación subjetiva del estrés laboral y finalmente se revisan los estudios que exploran la medición de biomarcadores y estrés laboral en médicos residentes.

Se realizó búsqueda de literatura en las bases de datos EBSCO, Science direct, Medline a través de Pubmed y Google académico. Se utilizaron los términos DeCS: estrés laboral, citoquinas, medicina, cortisol, biomarcadores. Términos MeSH: burnout, citokynes, medicine, hydrocortisone, biomarkers. Se incluyeron investigaciones originales que evaluaron la relación entre el estrés laboral y biomarcadores, publicadas en idioma inglés, espańol y portugués. No se limitó la búsqueda respecto a las fechas de publicación de las investigaciones. La última búsqueda se realizó el 15 de Abril de 2019.

\section{CAMBIOS FISIOLÓGICOS ASOCIADOS AL ESTRÉS}

Dentro de las posibles clasificaciones de estrés, está la basada en la línea de tiempo, la cual permite predecir en cierta forma los cambios fisiológicos esperados de acuerdo al periodo de exposición al estresor, esta clasificación establece dos categorías: estrés agudo y estrés crónico. El primero es una respuesta física inmediata frente a un único evento traumático, la respuesta es transitoria con fines protectores y adaptativos (22-24).

En relación con el estrés agudo, se ha identificado una fase de reacción de alarma en la que se presenta una descarga del sistema simpatoadrenomedular con la liberación de adrenalina y cortisol $(25,26)$. El estrés estimula al núcleo paraventricular del hipotálamo y al locus coeruleus en el tallo cerebral, generando como respuesta la liberación de factor liberador de corticotropina y vasopresina con la consecuente activación del eje hipotálamo-hipófisissuprarrenal y el eje simpaticomeduloadrenal (27, 28). Los glucocorticoides y catecolaminas secretadas se unen a los receptores de sus células diana, entre ellos las células inmunes como monocitos y neutrófilos, en donde inhiben la secreción de citoquinas inflamatorias como interleuquina $1 \beta$ (IL-1 $\beta$ ), interleuquina 6 (IL-6), factor de necrosis tumoral alfa (TNF $\alpha$ ), interferón gama (INF- $\gamma$ ), y favorecen aquellas con efectos antiinflamatorios secretadas por linfocitos como: interleuquina 4 (IL4), interleuquina 10 (IL-10) e interleuquina 13 (IL-13) (29). En el estrés crónico, en el cual se incluye el estrés laboral (30), los estresores permanecen estables en el tiempo y el individuo desconoce cuándo terminará el desafío o está seguro de que esto no ocurrirá, y por lo general hay impacto en los roles sociales (22). Si un determinado estresor persiste, el eje hipotálamo-hipófisis-suprarrenal se fatiga, y se genera resistencia a los glucocorticoides, cambios que finalmente ocasionan la reactivación de las vías de transcripción inhibidas inicialmente, explicándose de esta manera el aumento de la secreción y concentración de las citoquinas proinflamatorias y la disminución de las antiinflamatorias, y a su vez múltiples condiciones patológicas como depresión, diabetes mellitus, osteoporosis, enfermedades autoinmunes, procesos neoplásicos y también se ha relacionado con el empeoramiento de otras patologías (29-31); por ejemplo, las infecciones de tracto respiratorio superior son más severas en las personas con mayor estrés psicológico, en quienes la concentración de IL-6 es más alta comparado con la población sana (32). Se ha descrito que la respuesta del organismo, específicamente del eje hipotálamo-hipófisis-suprarrenal, a cualquier tipo de estresor independientemente del agente causal es la misma (33). Debido a los cambios que sufren algunos productos de los ejes fisiológicos en situaciones estresantes y que pueden seńalar etapas del estrés mismo, se les ha denominado biomarcadores, dentro de los más estudiados se encuentran el cortisol y las citoquinas $(10,20)$.

Al ser el estrés un activador del eje hipotálamo-hipófisissuprarrenal, provoca la liberación de cortisol, que es una neurohormona con acciones vitales de carácter antiinflamatorio, inmunosupresor y homeostático; de modo que ante situaciones estresantes la concentración basal de ésta tiende a elevarse inmediatamente (34). El cortisol, es una molécula lipídica que circula unida a una proteína transportadora principalmente, utilizando la globulina, también conocida como transcortina, que es sintetizada en el hígado; adicionalmente en un porcentaje de $15 \%$ del 
cortisol es transportado unido a la albúmina que también es de síntesis hepática que funciona como un reservorio circulante que asegura su entrega a diferentes tejidos y una fracción menor, se encuentra no unido a proteínas denominada fracción libre de cortisol. El valor normal del cortisol plasmático es de $375 \mathrm{nmol} / \mathrm{L}$ y varía según el horario en el que se tome (35); sin embargo, el estrés físico, estrés emocional, los procesos patológicos inflamatorios e infecciosos afectan ese valor en cualquier fluido corporal y bajo cualquiera de éstas situaciones la concentración de cortisol aumenta $(34,36)$. El nivel de cortisol (sanguíneo, urinario o salival) es un buen marcador de la respuesta fisiológica ante diversas situaciones estresantes, inclusive se han encontrado diferencias entre escenarios como la jornada laboral y los tiempos libres o de descanso; observándose en algunos estudios una asociación directa y más confiable con la medición de los niveles de cortisol en saliva (37).

Por su parte las citoquinas son proteínas secretadas por diferentes tipos de células, entre las que se mencionan: leucocitos, células endoteliales y células del sistema nervioso como astrocitos y microglia, con efectos estimuladores o inhibitorios no solamente en la respuesta inflamatoria que busca eliminar cualquier tipo de noxa, sino también en procesos hematopoyéticos (35). En experimentos realizados en animales y en humanos se ha comprobado que las situaciones de estrés agudo y crónico generan cambios en sus concentraciones (36), sin embargo, los resultados entre los diferentes estudios suelen ser contradictorios, por lo que la teoría de Tian y cols (2014) en la que plantean cambios en la concentración de citoquinas ante el estrés como un proceso dinámico, debería tenerse en cuenta al momento de la medición e interpretación (29), adicionalmente otras situaciones podrían explicar dicha discordancia, como la definición poco clara de estrés (22, 39), el tipo, la duración y la intensidad del agente estresor y los métodos de medición de citoquinas usados (29). Antes la medición de citoquinas circulantes era limitada, pero con el advenimiento de nuevas tecnologías como los paneles de citoquinas múltiples, es factible la determinación de varias de ellas con una muestra de sangre $(40,41)$.

\section{ESTRÉS LABORAL}

Respecto al origen del estrés, se han descrito varios agentes causales, denominados agentes estresores; entre ellos, las situaciones laborales negativas, el encarcelamiento, las pruebas académicas, la pérdida de seres queridos, las dificultades financieras $(4,24,25,42)$. Agentes estresores como los altos niveles de responsabilidad, la carga de trabajo variable, conflictos laborales-familiares (interfaz hogar-trabajo), el pobre control del sitio de trabajo, las relaciones interpersonales, dificultades con los compańeros de trabajo y supervisores; favorecen el desarrollo de un tipo de estrés particular, denominado estrés laboral (43), con su exponente más severo el síndrome de Burnout, el cual cobra gran interés dado que la instauración de este tipo de estrés es gradual y lastimosamente una vez que se adquiere, no es posible retornar a un estado de normalidad (44).

\section{ESTRÉS LABORAL EN RESIDENTES MÉDICOS.}

Las residencias médicas se tratan de un sistema educativo que tiene por objeto completar la formación de los médicos en alguna especialización reconocida, mediante el ejercicio de actos profesionales de complejidad y responsabilidad progresivas. Durante este período de formación profesional de posgrado, que oscila entre 3 a 5 ańos, los médicos deben cumplir con actividades académicas y asistenciales o laborales (14). Varias investigaciones han referido que esta es una de las poblaciones más afectadas por situaciones de estrés tanto académico como laboral. El estrés académico se define como la angustia mental relacionada con el aprendizaje, dada por sentimientos de frustración y temor al fracaso académico. Se ha establecido que este tipo de estrés en situaciones normales genera un sentido de competencia y motivación por parte del estudiante que lo lleva a superar situaciones específicas con éxito; sin embargo, cuando no hay un buen manejo de las situaciones, puede transformarse en ansiedad y sentimientos de impotencia reflejados en el rendimiento académico, relaciones interpersonales y estado de salud $(3,40,45)$.

Por su parte el estrés laboral se describe como un estado avanzado o como parte de un estrés crónico en el contexto laboral, debido a situaciones que superan las capacidades del empleado (46).

Sibien los residentes médicos cumplen actividades deíndole laboral durante su período formativo y se consideran una población susceptible a desarrollar estrés laboral, debido a que presentan agotamiento emocional, sentimientos de agotamiento físico, falta de energía y estado de ánimo disminuido (15), en países como Colombia, pese a la ley No 1917 aprobada en Julio de 2018, aún se les reconoce como estudiantes, sin derecho a un salario (47). 


\section{MEDICIÓN SUBJETIVA DEL ESTRÉS LABORAL EN RESIDENTES MÉDICOS}

Para la medición subjetiva del estrés laboral en médicos residentes, se han utilizado instrumentos como el MBI-HSS, la cual mide tres componentes: agotamiento emocional, despersonalización y falta de logro personal. Esta escala se encuentra validada para Colombia $(48,49)$. El cuestionario breve de Burnout (CBB), que comprende tres componentes principales; los antecedentes, los factores y las consecuencias del síndrome de Burnout (50) y el CVP 35 (Calidad de vida profesional 35) que evalúa la carga en el trabajo, el apoyo directivo, la motivación intrínseca, la calidad de vida percibida y la motivación al terminar la jornada laboral (51).

\section{BIOMARCADORES Y ESTRÉS LABORAL EN MÉDICOS RESIDENTES}

Entre los biomarcadores que se han estudiado en relación con el Burnout se encuentran; el cortisol, la hormona adrenocorticotropa (ACTH), la proteína de unión al calcio (S100B), el perfil metabólico y la distribución leucocitaria.

En 1991 Coeck y cols incluyeron ocho residentes del hospital de Middelheim en Estados Unidos, se evaluaron dos momentos; el primero en un turno de 24 horas seguidas y el segundo en un turno habitual (control), en cada uno de estos momentos se hicieron tres mediciones de cortisol y ACTH ( 9 am, 9 pm y 9 am) y se compararon. Solo se encontró diferencias estadísticamente significativas en los niveles de estos biomarcadores ( $p<0.02$ para cortisol y $\mathrm{p}<0,05$ para ACTH) al completar las 24 horas de turno (52).

Chatterton y Dooley (1999), evaluaron a seis residentes en quienes se midieron los niveles de testosterona plasmática, hormona luteinizante, cortisol y prolactina. Las mediciones se realizaron en 4 momentos, un día durante las dos primeras semanas de su residencia, un día inmediatamente después de un periodo de vacaciones, un día después del turno nocturno, y un día de turno habitual diurno, se realizaron mediciones en la mańana y en la tarde, y se compararon con los resultados obtenidos tras la medición de estrés psicológico.Se encontraron altos niveles de estrés en el primer y tercer día de medición, con una relación inversa entre estrés auto percibido y los niveles de testosterona plasmática $(\mathrm{p}<0.001)$ y hormona luteinizante $(\mathrm{p}<0.039)$, además de interrupción en el patrón normal de los niveles plasmáticos de cortisol $(\mathrm{p}<0.019)$, debido a los niveles de estrés expresados posterior a una noche de turno (53).
En 2014 Perdomo y cols evaluaron 94 residentes (turnos de 17 a 24 horas), en el Hospital Universitario Fundación Jiménez Díaz y el Hospital Universitario de Móstoles de Espańa, para determinar la relación entre estrés percibido y cortisol en sangre, número de distribución de fórmula leucocitaria, glucosa, colesterol total, LDL-colesterol, HDL-colesterol, triglicéridos y hormonas tiroideas. Las mediciones se realizaron a las $10 \mathrm{am}$ tras salir del turno. En el análisis de regresión logística solo se encontró diferencias estadísticamente significativas entre estrés percibido y la presencia de ansiedad y depresión (OR: 1.447, p<0.007), no se encontraron diferencias significativas respecto a los biomarcadores (54).

Eisenach y cols realizaron un ensayo piloto en 2015 con dieciocho médicos internos que ingresaron a residencia, estos fueron evaluados en 3 momentos diferentes; antes de empezar la residencia, al primer mes de la residencia y la tercer evaluación entre el tercer y quinto mes de residencia, se evaluaron los cambios en los biomarcadores antes y después de someter a los residentes a situaciones de estrés mental. Los biomarcadores incluidos fueron la proteína $\mathrm{C}$ reactiva, el cortisol y las catecolaminas. Respecto a los niveles séricos de cortisol, se encontraron cambios en el momento 1 de la medición $(7.01 \pm 1.66$ a $8.36 \pm 2.46)$ y en el seguimiento $2(7.01 \pm 1.80$ a $6.53 \pm 1.96)$, pero los resultados no fueron concluyentes (55).

Un estudio más reciente, publicado en 2016 por Gulen y cols, realizado en 48 residentes de medicina de emergencias de tres hospitales en Turquía, aplió escala de MBI, y se midió S100B, antes y después de un turno nocturno, encontrándose variaciones significativas en las dos mediciones y además, una correlación fuerte con los niveles de agotamiento emocional ( $\mathrm{p}<0.01)(56)$.

En la presente revisión de literatura no se encontraron estudios que evalúen la relación entre estrés laboral con el nivel de citoquinas en residentes médicos, a pesar que la teoría las describe como marcadores sensibles a las alteraciones fisiológicas desencadenadas en situaciones de estrés.

\section{CONCLUSIONES}

Los cambios fisiológicos asociados al estrés, justificarían el uso de biomarcadores como herramienta de medición objetiva, particularmente en condiciones de estrés crónico dado que se ha descrito que el eje hipotálamo-hipófisissuprarrenal se agota luego de una exposición prolongada al 
mismo agente estresor, con la consecuente alteración en los niveles de sustancias como las citoquinas proinflamatorias.

Las condiciones laborales adversas se han mencionado como desencadenantes de estrés crónico, razón por la cual los médicos residentes son considerados una población vulnerable; con variaciones según horas dedicadas a las actividades teóricas y a las actividades prácticas, horas de descanso y tipo de especialidad en la cual reciban preparación. El estudio de dicha condición en esta población se ha limitado a la valoración subjetiva y a través de la presente revisión se identificaron pocos estudios enfocados en explorar el uso de biomarcadores que permitan mediciones más precisas y que puedan utilizarse con fines diagnósticos en el ámbito de la salud ocupacional.

Se requieren investigaciones que examinen la relación entre estrés laboral y biomarcadores, en poblaciones como los residentes médicos, no solo porque esto apoyaría la toma de decisiones en pro del mejoramiento de las condiciones de salud de esta población, sino porque los niveles altos de estas sustancias también se relacionan con otras condiciones crónicas como depresión, enfermedad cardiovascular, enfermedades autoinmunes y cáncer.

\section{CONTRIBUCIÓN DE LOS AUTORES.}

JPBO: Concepción del proyecto original, Planificación del estudio, obtención de datos, interpretación de los resultados y redacción del manuscrito.

JPSS: Concepción del proyecto original, Planificación del estudio, obtención de datos, interpretación de los resultados y redacción del manuscrito.

AYMM: Planificación del estudio, obtención de datos, interpretación de los resultados y redacción del manuscrito.

\section{REFERENCIAS}

1. Chrousos GP. Stressors, stress, and neuroendocrine integration of the adaptive response: The 1997 Hans Selye Memorial Lecture. Annals of the New York Academy of Sciences. 1998;851(1):311-35.

2. Goodnite PM, editor Stress: a concept analysis. Nursing forum; 2014: Wiley Online Library.

3. Ali M, Asim H, Edhi AI, Hashmi MD, Khan MS, Naz F, et al. Does academic assessment system type affect levels of academic stress in medical students? A cross-sectional study from Pakistan. Medical education online. 2015; 20(1):27706.

4. Herrera Julian A. Psiconeuroinmunología para la práctica clínica. Primera edición. Santiago de Cali: Programa Editorial Universidad del Valle, 2009. 208 p.

5. Kopp MS, Thege BK, Balog P, Stauder A, Salavecz G, Rózsa $\mathrm{S}$, et al. Measures of stress in epidemiological research. Journal of Psychosomatic Research. 2010;69(2):211-25.

6. Antúnez Z, Vinet EV. Escalas de depresión, ansiedad y Estrés (DASS-21): Validación de la Versión abreviada en Estudiantes Universitarios Chilenos. Terapia psicológica. 2012;30(3):49-55.

7. Pedrero-Pérez EJ, de León JMR-S, Lozoya-Delgado P, RojoMota G, Llanero-Luque M, Puerta-García C. La "Escala de estrés percibido": estudio psicométrico sin restricciones en población no clínica y adictos a sustancias en tratamiento. Psicología conductual. 2015;23(2):305-24.

8. Maslach C, Schaufeli WB, Leiter MP. Job burnout. Annual review of psychology. 2001;52(1):397-422.

9. Hänsel A, Hong S, Cámara RJ, Von Kaenel R. Inflammation as a psychophysiological biomarker in chronic psychosocial stress. Neuroscience \& Biobehavioral Reviews. 2010;35(1):115-21.

10. O'Donovan A, Hughes BM, Slavich GM, Lynch L, Cronin M-T, O'Farrelly C, et al. Clinical anxiety, cortisol and interleukin-6: Evidence for specificity in emotionbiology relationships. Brain, behavior, and immunity. 2010;24(7):1074-7.

11. Szabo YZ, Newton TL, Miller JJ, Lyle KB, FernandezBotran R. Acute stress induces increases in salivary IL10 levels. Stress. 2016;19(5):499-505.

12. Bellingrath S, Rohleder N, Kudielka BM. Healthy working school teachers with high effort-reward-imbalance and overcommitment show increased pro-inflammatory immune activity and a dampened innate immune defence. Brain, behavior, and immunity. 2010;24(8):1332-9.

13. Ebrahimi S, Kargar Z. Occupational stress among medical residents in educational hospitals. Annals of Occupational and Environmental Medicine. 2018;30(1):51.

14. Sarmiento PJ, Parra Chico A. Calidad de vida en médicos en formación de posgrado. Persona y Bioética. 2015;19(2):290-302.

15. Prins JT, Gazendam-Donofrio SM, Tubben BJ, Van Der Heijden FM, Van De Wiel H, Hoekstra-Weebers JE. Burnout in medical residents: a review. Medical education. 2007;41(8):788-800.

16. Catsicaris C, Eymann A, Cacchiarelli N, Usandivaras I. La persona del médico residente y el síndrome de desgaste profesional (burnout): Un modelo de prevención en la formación médica. Archivos argentinos de pediatría. 2007;105(3):236-40. 
17. Abdulghani HM, Al-Harbi MM, Irshad M. Stress and its association with working efficiency of junior doctors during three postgraduate residency training programs. Neuropsychiatr Dis Treat. 2015;11:3023-3029.

18. Fahrenkopf AM, Sectish TC, Barger LK, Sharek PJ, Lewin $\mathrm{D}$, Chiang VW, et al. Rates of medication errors among depressed and burnt out residents: prospective cohort study. BMJ. 2008;336(7642):488-91.

19. Nakata A. Psychosocial job stress and immunity: a systematic review. En: Psychoneuroimmunology. Qing Yan. Springer; 2012. p. 39-75.

20. Danhof-Pont MB, van Veen T, Zitman FG. Biomarkers in burnout: a systematic review. Journal of psychosomatic research. 2011;70(6):505-24.

21. Jonsdottir I, Sjörs A. Endocrine and immunological aspects of burnout: a narrative review. European journal of endocrinology. 2018;1(aop).

22. Segerstrom SC, Miller GE. Psychological stress and the human immune system: a meta-analytic study of 30 years of inquiry. Psychological bulletin. 2004;130(4):601.

23. Illera D. Síndrome de Burnout, aproximaciones teóricas. Resultado de algunos estudios en Popayán. Revista Facultad Ciencias de la Salud: Universidad del Cauca. 2006;8(3):21-9.

24. Botero Ceballos LE, Arboleda Amórtegui G, Gómez Fernández AM, García Cardona M, Agudelo Suárez AA. Depresión en personas recluidas en centros penitenciarios: revisión narrativa. Rev. Fac. Cienc. Salud Univ. Cauca [Internet]. 1 de enero de 2019;21(1):23-33. Disponible en: https://revistas.unicauca.edu.co/index. $\mathrm{php} / \mathrm{rfcs} /$ article/view/1259.

25. S Moscoso M. De la mente a la célula: Impacto del estrés en psiconeuroinmunoendocrinología. Liberabit. 2009;15(2):143-52.

26. Koelsch S, Boehlig A, Hohenadel M, Nitsche I, Bauer K, Sack U. The impact of acute stress on hormones and cytokines, and how their recovery is affected by music-evoked positive mood. Scientific Reports. 2016; 6:23008.

27. Herman JP, McKlveen JM, Ghosal S, Kopp B, Wulsin A, Makinson R, et al. Regulation of the hypothalamic-pituitaryadrenocortical stress response. Comprehensive Physiology. 2011;6(2):603-21.

28. McDevitt RA, Szot P, Baratta MV, Bland ST, White SS, Maier SF, et al. Stress-induced activity in the locus coeruleus is not sensitive to stressor controllability. Brain Research. 2009; 1285:109-18.

29. Rui Tian, Gonglin Hou, Dan Li, and Ti-Fei Yuan. A Possible Change Process of Inflammatory Cytokines in the Prolonged Chronic Stress and Its Ultimate Implications for Health. The Scientific World Journal. 2014; 2014: 8p.
30. Mariotti A. The effects of chronic stress on health: new insights into the molecular mechanisms of brain-body communication. Future Sci OA. 2015;1(3):FSO23.

31. Duric V, Clayton, S., Leong, M. L., Yuan, L. L. Comorbidity factors and brain mechanisms linking chronic stress and systemic illness. Neural plasticity. 2016; 2016.

32. Kunz-Ebrecht SR, Mohamed-Ali V, Feldman PJ, Kirschbaum C, Steptoe A. Cortisol responses to mild psychological stress are inversely associated with proinflammatory cytokines. Brain, behavior, and immunity. 2003;17(5):373-83.

33. Elenkov IJ, Chrousos GP. Stress hormones, proinflammatory and antiinflammatory cytokines, and autoimmunity. Annals of the New York Academy of Sciences. 2002;966(1):290-303.

34. Guerrero Julia. Para entender la acción de cortisol en inflamación aguda: una mirada desde la glándula suprarrenal hasta la célula blanco. Rev. méd. Chile. 2017; $145(2)$ ): 230-239.

35. Abbas AK, Lichtman AH, Pillai S. Basic immunology: functions and disorders of the immune system: Elsevier Health Sciences; 2014.

36. Torpy DJ, \& Ho, J. T. Value of free cortisol measurement in systemic infection. Hormone and Metabolic Research. 2007;39(06):439-44.

37. Fajardo-Zapata ÁL, Montejo-Angel F, Molano-Vargas G, Hernández-Nińo J, Quintero-Guzmán A. Correlación entre los Factores de Riesgo Intralaboral y los Niveles Séricos de Cortisol en Docentes Universitarios. Ciencia \& trabajo. 2013;15(46):1-6.

38. 1. Do Yup Lee, Eosu Kim, Man Ho Choi. Technical and clinical aspects of cortisol as a biochemical marker of chronic stress. BMB reports. 2015;48(4):209-16.

39. 1. Delgado Quińones GP, Gómez Naranjo HJ, Jaramillo Gutiérrez M, Maya Giraldo P, Narváez Reyes MI, Salazar Pajoy AC, et al. Estudio descriptivo sobre estrés en estudiantes de medicina de la Universidad de Manizales. Archivos de Medicina. 2014;(11):37-45.

40. Kamezaki Y, Katsuura S, Kuwano Y, Tanahashi T, Rokutan K. Circulating cytokine signatures in healthy medical students exposed to academic examination stress. Psychophysiology. 2012;49(7):991-7.

41. Richens JL, Urbanowicz RA, Metcalf R, Corne J, O'Shea P, Fairclough L. Quantitative validation and comparison of multiplex cytokine kits. Journal of biomolecular screening. 2010;15(5):562-8.

42. Anderson Norman B. JSB, Belar Cynthia D.,Breckler Steven J. Stress in America: Our Health at Risk American Psychological Association. 2012:73.

43. Baka $七$, Bazińska R. Polish adaptation of three selfreport measures of job stressors: the Interpersonal Conflict at Work Scale, the Quantitative Workload 
Inventory and the Organizational Constraints Scale. Int J Occup Saf Ergon. 2016; 22(1):32-9.

44. Vega NV, Sanabria A, Domínguez LC, Osorio C, Ojeda $\mathrm{C}$, Bejarano M. Síndrome de desgaste profesional en cirujanos colombianos. Revista Colombiana de Cirugía. 2009;24(3):153-64.

45. García NB, Zea RM. Estrés académico. Revista de psicología Universidad de Antioquia. 2012;3(2):55-82.

46. Navinés R, Martín-Santos R, Olivé V, Valdés M. Estrés laboral: implicaciones para la salud física y mental. Medicina Clínica. 2016; 146:359-366.

47. Organización Panamericana de la Salud - Área de Sistemas de Salud basados en la Atención Primaria de Salud. Residencias médicas en América Latina. Washington D.C.: OPS; 2011. 127p

48. Córdoba L, Tamayo JA, González MA, Martínez MI, Rosales A, Barbato SH. Adaptation and validation of the Maslach Burnout inventory-human services survey in Cali, Colombia. Colombia Médica. 2011;42(3):286-93.

49. Hederich-Martínez C, Caballero-Domínguez CC. Validation of Maslach Burnout Inventory-Student Survey (MBI-SS) in Colombian academic context. CES Psicología. 2016;9(1):1-15.

50. Dipp AJ, Gutiérrez R.V, Salas MEG, Flores JAT. Valoración del desempeńo docente y presencia de burnout en maestros de educación superior. Revista electrónica diálogos educativos. 2011; 21:65-80.

51. Rivera-Ávila DA, Rivera-Hermosillo JC, GonzálezGalindo C. Validación de los cuestionarios CVP-35 y MBI-HSS para calidad de vida profesional y burnout en residentes. Investigación en Educación Médica. 2017;6(21):25-34.

52. Coeck C, Jorens PG, Vandevivere J, Mahler C. ACTH and cortisol levels during residency training. $\mathrm{N}$ Engl J Med. 1991;325(10):738.

53. Chatterton Jr RT, Dooley SL. Reversal of diurnal cortisol rhythm and suppression of plasma testosterone in obstetric residents on call. Journal of the Society for Gynecologic Investigation. 1999;6(1):50-4.

54. Deschamps Perdomo A, Zabala R, Campo Bals M, Mahíllo Fernández I, Otero Borrego C. Estrés percibido por médicos residentes que realizan guardias de urgencia en dos hospitales de Madrid y determinación de biomarcadores de estrés. Rev. Asoc. Esp. Espec. Med. Trab. 2014; 23(1): 238-245.

55. Eisenach JH, Sprung J, Clark MM, Shanafelt TD, Johnson BD, Kruse TN, et al. The Psychological and Physiological Effects of Acute Occupational Stress in New Anesthesiology Residents. A Pilot Trial. The Journal of the American Society of Anesthesiologists. 2014;121(4):878-93.
56. Gulen B, Serinken M, Eken C, Karcıoglu Ö, Kucukdagli OT, Kilic E, et al. Serum S100B as a Surrogate Biomarker in the Diagnoses of Burnout and Depression in Emergency Medicine Residents. Academic Emergency Medicine. 2016;23(7):786-9. 